\title{
Las actividades turísticas en la economía colaborativa ¿en qué consisten y quiénes son sus proveedores?
}

\author{
Santiago Melián González* Jacques Bulchand Gidumal** \\ Universidad de Las Palmas de Gran Canaria (España)
}

\begin{abstract}
Resumen: La economía colaborativa sigue expandiéndose en el sector turístico. Aunque ya existía desde hace tiempo, la oferta de actividades turísticas en este ámbito está adquiriendo una mayor relevancia debido a la irrupción de Airbnb. En este artículo aportamos información detallada del tipo de actividades turísticas que se oferta, se compara con las actividades existentes en dos plataformas de actividades profesionales, y se analizan los proveedores teniendo en cuenta lo que la literatura de la economía colaborativa establece al respecto. Se descargaron los datos de Airbnb de cuatro comunidades autónomas españolas y se analizaron de manera cuantitativa y cualitativa. Los resultados reflejan que la economía colaborativa y la economía tradicional comparten similitudes en su oferta de actividades turísticas, a la vez que también presentan diferencias. Una buena parte de sus proveedores no tiene el perfil que tradicionalmente se les ha atribuido en la literatura sobre le economía colaborativa. A su vez, los responsables de la gestión de los destinos turísticos pueden estar tranquilos en lo referido a la percepción de sus visitantes, dado el alto nivel de satisfacción que muestran los mismos.
\end{abstract}

Palabras Clave: Tours; Actividades turísticas; Economía; Economía colaborativa.

Tourism in the sharing economy: what is it and who provides it?

Abstract: The sharing economy continues to grow inside the tourism industry. Although sharing tours and activities have been being offered now for quite some time, Airbnb has produced major changes in what is on offer. This articles compares what is on offer with the activities detailed in two webs of major professional suppliers to go on to contrast the suppliers' characteristics with the theory of the sharing economy as reflected in the literature available. AirBnB tours and activities were downloaded for four regions in Spain and analysed qualitatively and quantitatively allowing us to identify the "shared" activities with the traditional tourism economy and the differences. The conclusion reached is that most "sharing economy" suppliers do not fit the profile generally attributed to the same in the literature, while managers of DMOs can rest at ease with visitor perception since such is highly satisfied with the experiences and activities on offer.

Keywords: Museo; Tours; Tourism activities; Economy; Collaborative economy.

\section{Introducción}

Entre los elementos que determinan la competitividad de los destinos turísticos se encuentran los recursos y actividades que los turistas encuentran atractivos. Un ejemplo es la oferta de actividades en las que los visitantes participan de forma activa. Según Crouch y Ritchie (1999: 148) "la gama de actividades dentro de un destino representa uno de los aspectos más críticos del atractivo del destino".

Tradicionalmente, en los destinos turísticos, las actividades dirigidas a los visitantes se han ofertado por agentes formales, tales como guías oficiales, agencias de viajes y empresas locales. Sin embargo, y al igual que ha sucedido en la industria alojativa, la economía colaborativa (en adelante, EC) también ha penetrado en el campo de las actividades turísticas. No se trata de un fenómeno reciente, ya que, por ejemplo, ToursByLocals, una plataforma pionera en este campo, lleva más de diez años en funcionamiento. No obstante, recientemente se han dado una serie de fenómenos que han desencadenado el crecimiento

** Universidad de Las Palmas de Gran Canaria (España); E-mail: santiago.melian@ulpgc.es; https://orcid.org/0000-0002-7662-3154
*niversidad de Las Palmas de Gran Canaria (España); E-mail: jacques.bulchand@ulpgc.es; https://orcid.org/0000-0001-8522-2013 
exponencial de este tipo de oferta. Probablemente, uno de los más relevantes sea la popularización en el bienio 2015-2016 del gigante de la EC Airbnb.

La presencia de la EC en el turismo ha sido ampliamente investigada en los campos del alojamiento y transporte (e.g., Cramer y Krueger, 2016; Guttentag et al., 2018; Zervas, Proserpio y Byers, 2017; entre muchos otros). Recientemente, también se han realizado algunas investigaciones en el ámbito de las experiencias gastronómicas (Ketter, 2019). Sin embargo, en el caso de las actividades turísticas apenas hay investigación, por lo que el contenido y características de las mismas son desconocidos. En este sentido, Bryon (2012) incluyó a guías no oficiales, que operaban en plataformas de Internet, en una investigación cualitativa sobre diferentes tipos de guías turísticos. En una plataforma de EC de actividades turísticas, Melián-González y Bulchand-Gidumal (2018a) descubrieron que la mayoría de las actividades realizadas consistían en tours guiados. Por su parte, Batle, Garau-Vadell y Orfila-Sintes (2019) estudiaron factores explicativos de ser un proveedor de actividades turísticas en el campo de la EC.

Las experiencias de los turistas en los destinos influyen en su imagen del mismo (Beerli y Martin, 2004). La mayoría de los estudios que explican el éxito de los destinos turísticos (e.g., Crouch \& Ritchie, 1999, 2000) suponen que sus responsables pueden influir en estas experiencias, así como en el conjunto de actividades que los turistas encuentran en los destinos. Esta influencia se puede lograr a través de la regulación, la educación, los incentivos, reuniones con representantes y/o acciones de formación. Sin embargo, en teoría, la EC se basa en la iniciativa de particulares, por lo que su actividad puede resultar difícil de influir por aquellas acciones que tradicionalmente están dirigidas a agentes formales o profesionales. Por lo tanto, una parte de la oferta turística podría estar fuera del alcance de los proyectos de las organizaciones de gestión de destinos turísticos (Destination Management Organization, en adelante, DMO).

Por otro lado, varios autores afirman que las actividades turísticas del campo de la EC, por sí mismas, pueden aumentar el atractivo de los destinos. Ello se debe a que los visitantes quieren conocer a residentes locales y obtener autenticidad social (Batle, Garau-Vadell y Orfila-Sintes, 2019; Dredge y Gyimóthy, 2015; Paulauskaite, Powell, Coca- Stefaniak y Morrison, 2017). Este planteamiento supone que aquellos que ofrecen actividades turísticas son locales aficionados, en lugar de proveedores tradicionales. Por lo tanto, a partir de la idea de que la EC representa una nueva forma de consumo, se cree que las actividades turísticas de la EC pueden ser un nuevo tipo de oferta turística. El motivo es que sus proveedores, particulares locales, pueden proporcionar una oferta diferente (e.g., más personalizada o auténtica) de la que ofrecen los proveedores tradicionales. Un debate similar, y sin resolver, se ha producido en la industria del alojamiento. Existe evidencia de que el alojamiento colaborativo puede ser muy similar al alojamiento en hoteles (e.g., Guttentag \& Smith, 2017), pero también hay resultados que reflejan que, en algunos casos, hay diferencias (e.g., Blal, Singal y Templin, 2018).

No obstante, y a pesar de la relevancia que la EC tiene en estos momentos, apenas existe investigación sobre lo que está ocurriendo con una de sus manifestaciones más recientes como son las actividades turísticas. El objetivo de este trabajo es analizar las actividades turísticas ofertadas en el entorno de la EC. Algunas de las preguntas a contestar son: ¿qué tipo de actividades incluyen? ¿Son equivalentes a las que se ofrecen en las plataformas orientadas a proveedores profesionales? ¿Son realmente ofrecidas por particulares? Y ¿están satisfechos los consumidores? Con ese fin, hemos seguido una metodología estructurada en dos etapas. Primero, descargamos y analizamos la oferta de actividades turísticas de Airbnb en cuatro importantes destinos turísticos españoles. Esta oferta se comparó con la de dos importantes plataformas en las que los proveedores son profesionales (B2C). En segundo lugar, y para comprender mejor las características de los proveedores de actividades turísticas en plataformas de la EC, realizamos entrevistas semiestructuradas con proveedores profesionales que ofrecen experiencias en una plataforma de EC en uno de los destinos.

La contribución de este artículo consiste en aportar información sobre una faceta de la EC, en el ámbito turístico, apenas estudiada. El análisis se hace en el marco de las premisas en las que se sustenta la EC, de manera que los resultados permiten contrastar algunos de los razonamientos o argumentos que suelen usarse para valorar la contribución de este tipo de actividad. El artículo está estructurado de la siguiente manera: la primera sección aborda la revisión de la literatura e incluye las preguntas de investigación, la siguiente explica la metodología, luego se describen los resultados y, para finalizar, se presentan la discusión, las conclusiones y las limitaciones.

\section{Literatura}

Aparte de cuestiones básicas, como las infraestructuras y la hospitalidad de la población, los destinos turísticos se basan en una oferta variada de productos turísticos (Buhalis, 2000). Las actividades turísticas forman parte de la misma. Según Ritchie y Crouch (2003), las DMO deben asumir un papel 
activo respecto a lo que los destinos ofrecen a los visitantes. En este sentido, Heath y Wall (1992), Presenza, Sheehan y Ritchie (2005), Sainaghi (2006) y Müller y Berger (2012) atribuyen funciones concretas a las DMO, como la formulación de estrategias, el desarrollo de productos, la capacitación, y la supervisión de la calidad del servicio. Estos trabajos suponen que quienes ofrecen las actividades turísticas son profesionales, empresas u otras organizaciones formales. Sin embargo, como ha ocurrido en el alojamiento y en el transporte, la EC ha traído un nuevo tipo de proveedor de actividades turísticas: particulares que actúan como aficionados. Esta oferta apenas ha sido analizada en la literatura científica.

\subsection{Las actividades turísticas en la EC}

Una de las premisas de la EC es que las personas tienen recursos infrautilizados que pueden ser aprovechados por otras personas (Botsman y Rogers, 2011; Frenken y Schor, 2017; Schor y Attwood-Charles, 2017). Por lo tanto, las plataformas de la EC fomentan los intercambios entre pares (P2P) y permiten que los particulares participen en negocios turísticos tradicionales como el alojamiento y el transporte. Ambos tipos de proveedores, profesionales y particulares, coexisten y ofrecen servicios a los visitantes. En el ámbito de las actividades turísticas, los tours guiados, el turismo deportivo y el turismo de aventura, que tradicionalmente han sido ofrecidos por profesionales y empresas (Bryon, 2012; Buckley, 2007; Kurtzman, 2005), están también presentes ahora en el campo de la EC.

Plataformas de EC pioneras en las actividades turísticas fueron ToursByLocals y Trip4real. Airbnb adquirió esta última en 2016. A diferencia del caso del alojamiento colaborativo y, más precisamente, de la plataforma Airbnb, apenas hay datos sobre el contenido de las actividades turísticas proporcionadas a través de la EC. A partir de datos de Trip4real, sobre varios destinos urbanos, Melián-González y Bulchand-Gidumal (2018a) encontraron los siguientes tipos de actividades: rutas (62,59\%), gastronomía $(20,58 \%)$, deportes y aventura (8,33\%), excursiones y excursiones de un día (4,93\%), talleres $(1,19 \%)$, vida nocturna $(1,19 \%)$ y naturaleza y descanso $(1,19 \%)$. Hay que tener en cuenta que estos resultados provienen de una plataforma de alcance limitado, que actualmente no está disponible (ya que fue adquirida por Airbnb), del año 2016, y antes de la reciente expansión del sector. Por lo tanto, planteamos la siguiente pregunta de investigación.

Pregunta de investigación 1: ¿qué tipo de actividades turísticas se ofertan en la EC?

Aunque no es concluyente, existe evidencia sobre la equivalencia entre los servicios de la EC y los servicios tradicionales en el sector turístico. Así, si bien hay trabajos que han encontrado que ambos servicios son equivalentes y, por ello, uno actúa como sustituto del otro, otros trabajos han hallado una coexistencia complementaria. Por ejemplo, Zervas et al. (2017) descubrieron que el aumento en la oferta de Airbnb afectaba negativamente los ingresos de los hoteles económicos. Guttentag y Smith (2017) encontraron que casi dos tercios de los huéspedes de Airbnb habían usado la plataforma como sustituto de un hotel, especialmente en el caso de hoteles de gama media. Benítez-Aurioles (2019) descubrió que Airbnb había tenido un impacto negativo en hoteles de todas las categorías. Blal, Singal y Templin (2018) encontraron resultados mixtos. Si bien descubrieron que la oferta de propiedades en Airbnb no afectaba las ventas de hoteles, lo que sugeriría que el alojamiento colaborativo complementa la industria hotelera, los autores también encontraron que había una relación negativa entre la satisfacción de los huéspedes de Airbnb y las ventas de hoteles, lo que indica un efecto de sustitución. Gyódi (2019) descubrió que la sustituibilidad entre los hoteles y Airbnb dependía de la ubicación, porque, aunque a nivel mundial había un alto grado de sustituibilidad, la oferta de Airbnb complementaba la industria hotelera en las áreas suburbanas. En el caso del transporte, Berger, Chen y Frey (2018) encontraron que la expansión de Uber ha reducido las ganancias potenciales de los conductores de servicios de taxi convencionales.

En el caso de las actividades turísticas, Airbnb afirma que los proveedores son locales dispuestos a compartir sus conocimientos y habilidades de una manera diferente a la de la oferta tradicional. Como se ha dicho, algunos autores consideran que los visitantes desean conocer a locales que brinden experiencias auténticas (Batle, Garau-Vadell y Orfila-Sintes, 2019; Dredge y Gyimóthy, 2015; Paulauskaite, Powell, Coca- Stefaniak y Morrison, 2017). Al mismo tiempo, Airbnb destaca la oportunidad que tienen estos locales de obtener ingresos y que pueden lanzar un negocio basado en su plataforma. Por lo tanto, se pide a los anfitriones que cumplan un doble papel: locales que ofrecen actividades novedosas basadas en sus conocimientos y habilidades, y empresarios que pueden desarrollar un negocio a partir de la plataforma. 
Sin embargo, los hallazgos comentados, respecto a las industrias alojativas y del transporte, sugieren que en las plataformas de EC podrían estarse ofreciendo servicios prestados por las empresas turísticas tradicionales. A su vez, esos resultados reflejan que la EC también puede funcionar como un complemento de estas últimas. La falta de investigación hace que sea difícil saber qué sucede en la industria de las actividades turísticas. Por lo tanto, planteamos las siguientes dos preguntas de investigación.

Pregunta de investigación 2: ¿la oferta de actividades turísticas en la EC se solapa con la oferta de negocios turísticos?

Pregunta de investigación 3: ¿la oferta de actividades turísticas en la EC se basa en características no profesionales?

\subsection{Proveedores en la EC}

Apte y Davis (2019) explican que la EC se concibió inicialmente para que los particulares ofrecieran productos y servicios. De esta manera, Schor (2014) y Melián-González (2018) afirman que los defensores de la EC declaran que representa una oportunidad para que cualquiera pueda obtener ingresos económicos. De hecho, la EC no está restringida a profesionales, como sí ocurre con los canales de distribución tradicionales. Esto implica que la EC es una alternativa en la que cualquiera puede compartir sus recursos infrautilizados, lo que puede generar una compensación monetaria. De hecho, muchas plataformas de la EC no exigen ningún tipo de requisito profesional y/o académico a las personas que se unen a ellas.

Sin embargo, a medida que se expandía la EC, algunos autores afirmaron que quienes ofrecían productos y servicios no actuaban como aficionados, sino que resultaba evidente que, en vez de compartir, estaban trabajando (Aloisi, 2016; Frenken \& Schor, 2017; Schor, 2017). Además, varias instituciones y autores declararon que la EC también estaba dirigida a microempresarios (Sundarajaran, 2014; Comisión Europea, 2016; Zhang, Bufquin y Lu, 2019). Este posible escenario de trabajadores y emprendedores podría ser contrario a la premisa de que los destinos turísticos pueden aumentar su competitividad a partir de particulares locales que ofrecen actividades turísticas auténticas (Batle, Garau-Vadell y Orfila-Sintes, 2019).

La EC abarca diferentes tipos de intercambios y plataformas (Bulchand-Gidumal \& Melián-González, 2017). Existen plataformas con ánimo de lucro (e.g., Airbnb, Uber, TaskRabbit y Upwork) en las que las personas perciben ingresos, por lo que se sientan las bases para que pueda comenzarse a hablar de profesionales. En otras, las personas no buscan ganancias sino compartir costes (e.g., BlaBlaCar). Sin embargo, incluso en plataformas con fines de lucro puede ser difícil afirmar que todas las personas actúan como profesionales. Por otro lado, puede ser que el amateurismo no sea factible en estas plataformas, ya que los consumidores de la EC valoran la profesionalidad y los servicios excelentes (Melián-González, 2018). En este sentido Zhang, Bufquin y Lu (2019) consideran directamente a los anfitriones de Airbnb como microempresarios. Sin embargo, Gardiner (2018) descubrió que los consumidores esperaban más autenticidad cuando una actividad turística se proporcionaba a través de Airbnb. Por el contrario, si la actividad era ofrecida por una empresa turística, los consumidores valoraban la cualificación de los instructores.

Existe evidencia respecto a la existencia de profesionales en la EC, específicamente en el caso de Airbnb. En Chicago, Li, Moreno y Zhang (2015) encontraron que en Airbnb, el 18\% de los anfitriones eran profesionales (i.e., aquellos que ofrecían más de una propiedad). En España, Bulchand-Gidumal, Melián-González y López-Valcárcel (2019) encontraron que el 42,5\% de las propiedades pertenecían a anfitriones que ofrecían más de una propiedad. Sin embargo, estos autores consideraron profesionales a aquellos que ofrecían tres o más propiedades, lo que representaba el 18,8\% de las propiedades. Gyódi (2019) considera profesionales a aquellos que ofrecen una o más propiedades, mientras que supone no profesionales a los que alquilan habitaciones. Con esta clasificación, el número de profesionales en la plataforma varía de 89,9\% en Varsovia a 50,5\% en Berlín. Los profesionales también operan en el caso de Uber, dado que un tercio de los conductores cuya única ocupación era conducir a través de Uber (38\%) conducía más de 35 horas por semana (Hall y Krueger, 2015).

Es importante saber quién ofrece las actividades turísticas en la EC. Hasta ahora, los académicos y los responsables de las DMO han supuesto que los profesionales y las empresas son los que lo hacen. Este tipo de proveedor debe tener en cuenta algún tipo de regulación (e.g., licencia profesional, responsabilidad civil, normativa de actividades económicas) que busca garantizar la calidad de los servicios ofrecidos y proteger a los consumidores. Sin embargo, este puede no ser el caso cuando las personas ofertan 
actividades de una manera no profesional en plataformas de EC. Por otro lado, este perfil teóricamente no profesional se considera una forma para que los destinos aumenten su competitividad. Sin embargo, se ha encontrado que aquellos que brindan servicios turísticos a través de plataformas de EC pueden considerarse profesionales. Si este es el caso, surge la pregunta sobre por qué estos usan una plataforma de EC. Por lo tanto, planteamos las siguientes dos preguntas de investigación.

Pregunta de investigación 4: ¿qué tipo proveedores de actividades turísticas predominan en la EC, profesionales o aficionados?

Pregunta de investigación 5: ¿por qué los proveedores profesionales usan la EC para ofrecer sus actividades?

\section{Metodología}

Aunque en sus inicios Airbnb únicamente se orientaba a la oferta de alojamiento colaborativo, en los últimos años ha ido ampliando sus ámbitos de actuación, incluyendo desde 2016 los apartados de experiencias (Airbnb Experiences) y aventuras (Airbnb Adventures). Este estudio se basa en datos de Airbnb Experiences porque, hasta donde sabemos, es la plataforma de EC más importante en cuanto a la oferta de actividades turísticas. La metodología aplicada tiene dos etapas. Primero, seguimos un enfoque cuantitativo mediante la descarga de las actividades turísticas ofrecidas en la citada plataforma. Posteriormente, realizamos una investigación cualitativa basada en, por una parte, el análisis de contenido del texto que los proveedores usan para presentarse y, por otra, en entrevistas semiestructuradas.

\subsection{Etapa cuantitativa}

En el primer paso, se descargó manualmente, entre enero y marzo de 2019, la información sobre todas las actividades turísticas ofertadas en Airbnb en cuatro importantes destinos turísticos españoles. Estas regiones fueron Madrid, Cataluña, Islas Baleares e Islas Canarias. Dos de ellos (Madrid y Cataluña) son destinos en los que una ciudad es el principal atractivo (Madrid y Barcelona, respectivamente), mientras que los otros dos (Islas Baleares e Islas Canarias) son destinos de vacaciones de sol y playa. Según el Instituto Nacional de Estadística español, en 2019 estas regiones recibieron el 64,31\% del total de visitantes de España. La Tabla 1 muestra datos sobre la cantidad de actividades turísticas encontradas y el número de visitantes internacionales en cada una de las cuatro regiones.

Tabla 1: Características de las regiones analizadas

\begin{tabular}{|l|r|r|}
\hline \multicolumn{1}{|c|}{ Región } & Actividades & \multicolumn{2}{c|}{$\begin{array}{c}\text { Turistas internacionales } \\
\text { en 2019 }\end{array}$} \\
\hline Cataluña & 288 & 19.358 .203 \\
\hline Canarias & 187 & 13.147 .009 \\
\hline Madrid & 168 & 7.638 .375 \\
\hline Baleares & 87 & 13.680 .923 \\
\hline TOTAL & 730 & 53.824 .510 \\
\hline
\end{tabular}

Fuente: Airbnb e INE

Cuando los proveedores suben una actividad turística a Airbnb seleccionan etiquetas para describir el tipo de actividad que están ofreciendo. El rango de etiquetas es grande, lo que genera que existan actividades similares con diferentes etiquetas. En nuestra muestra, considerando todas las etiquetas que los anfitriones adjuntaron a sus actividades, había 62 etiquetas diferentes. Estas se agruparon en grupos homogéneos. Para ello, los autores utilizaron categorías generales (e.g., tours, talleres, deportes, excursiones, gastronomía) y trataron de incluir todas las actividades en las mismas. Estas categorías generales son las de las principales plataformas actualmente disponibles a través de las cuales las empresas y profesionales ofrecen sus actividades turísticas (e.g., GetYourGuide y Viator). 
Posteriormente, los autores crearon categorías adicionales cuando en cada una de las categorías generales había actividades que mostraban un contenido diferente. Por ejemplo, las actividades relacionadas con los tours se agruparon en tres categorías diferentes: tours arquitectónicos e históricos, tours temáticos, y excursiones y paseos. La Tabla 2 recoge las categorías definitivas de actividades y una descripción su contenido. La Tabla 3 recopila ejemplos de las actividades que incluye cada categoría.

\section{Tabla 2: Tipos de actividades}

\begin{tabular}{|l|l|}
\hline \multicolumn{1}{|c|}{ Categorías } & \multicolumn{1}{c|}{ Descripción } \\
\hline $\begin{array}{l}\text { Tours } \\
\text { arquitectónicos/ } \\
\text { históricos }\end{array}$ & $\begin{array}{l}\text { Tours guiados que buscan enseñar las características arquitectónicas, históricas, } \\
\text { y/o artísticas más importantes de un destino. }\end{array}$ \\
\hline Tours temáticos & $\begin{array}{l}\text { Tours guiados que llevan a los visitantes a participar en actividades de ocio } \\
\text { características de un destino tales como ruta por bares, lugares para sacar fotos, } \\
\text { galerías de arte, moda, o tiendas. }\end{array}$ \\
\hline Excursiones/paseos & $\begin{array}{l}\text { Paseos largos y excursiones en diferentes medios de transportes ambos en un } \\
\text { entorno natural. }\end{array}$ \\
\hline Talleres & $\begin{array}{l}\text { Diferentes tipos de actividades en las que las personas aprenden habilidades } \\
\text { físicas o psicológicas }\end{array}$ \\
\hline Deportes & Practicar una actividad deportiva. \\
\hline $\begin{array}{l}\text { Enseñanza de } \\
\text { deportes }\end{array}$ & Clases para aprender un tipo de deporte. \\
\hline Visitas & Visitas guiadas a lugares como museos, viñedos, granjas, o mercados \\
\hline Gastronomía & Actividades en las que los participantes prueban bebidas y/o comidas especiales. \\
\hline Música/baile & Eventos relacionados con la música o el baile. \\
\hline Socialización & Reuniones que buscan promover relaciones con otras personas. \\
\hline
\end{tabular}

Fuente: Elaboración propia

Tabla 3: Ejemplos de actividades ofrecidas en Airbnb en cada categoría

\begin{tabular}{|l|l|}
\hline \multicolumn{1}{|c|}{ Categorías } & \multicolumn{1}{c|}{ Actividades } \\
\hline $\begin{array}{l}\text { Tours arquitectónicos/ } \\
\text { históricos }\end{array}$ & $\begin{array}{l}\text { Tour por la ciudad en bicicleta eléctrica. Visitas a las zonas tradicionales } \\
\text { de la ciudad. }\end{array}$ \\
\hline Tours temáticos & $\begin{array}{l}\text { Tour fotográfico por el centro de la ciudad antigua. Las mejores tiendas } \\
\text { de artesanía local. }\end{array}$ \\
\hline Excursiones/paseos & Escalar y nadar en las montañas. Navegando y nadando en la costa. \\
\hline Talleres & Clases de cocina. Técnicas de fotografía urbana. \\
\hline Deportes & Bicicleta de montaña en pista de tierra. Buceo. Escalada. \\
\hline Enseñanza de deportes & Clases de surf en la playa. \\
\hline Visitas & $\begin{array}{l}\text { Visita guiada a una finca de plataneras. Descubriendo la permacultura } \\
\text { en una granja. }\end{array}$ \\
\hline Gastronomía & Cerveza artesana. Cocina extravagante. Degustación de vinos. \\
\hline Música/baile & $\begin{array}{l}\text { Experiencia musical. Descubre locales de música. Flamenco detrás del } \\
\text { escenario. }\end{array}$ \\
\hline Socialización & Conocer gente y diversión. Encuentro en una azotea privada. \\
\hline
\end{tabular}

Fuente: Elaboración propia a partir de Airbnb 
Con el fin de comparar el tipo de actividades turísticas que se ofrecen en la EC con el proporcionado por empresas y profesionales, se analizó la oferta de dos plataformas B2C. GetYourGuide y Viator son dos de las plataformas más importantes mediante las que empresas y profesionales ofrecen sus actividades. Entre abril y mayo de 2019, los autores analizaron el contenido de estas dos plataformas en los destinos turísticos considerados y clasificaron las actividades encontradas en las mismas en las categorías descritas en la Tabla 2. Como se verá en la sección de resultados, no todas las actividades encontradas en ambas plataformas pudieron clasificarse en las categorías de la Tabla 2.

\subsection{Etapa cualitativa}

Para averiguar si las actividades turísticas en la EC se basan en atributos no profesionales de sus proveedores, se analizaron las autodescripciones que los mismos muestran en Airbnb. Se realizó un análisis de contenido de estas autodescripciones utilizando el software de análisis de contenido KH Coder. A tal efecto, a partir de la literatura, se asumió un enfoque deductivo y se establecieron dos categorías principales para el proceso de codificación (Saldaña, 2013). Una categoría se refiere a proveedores que destacan su perfil local, mientras que la otra se refiere a proveedores que enfatizan su perfil profesional.

A su vez, para determinar si aquellos que ofrecen actividades turísticas a través de Airbnb son empresas y profesionales o aficionados, consideramos la disponibilidad de las actividades durante la semana, concretamente en el periodo de tres meses posterior a la fecha de descarga de datos. La suposición es que aquellos que ofrecen sus actividades de manera irregular, o solo los fines de semana, podrían considerarse locales que combinan su oferta con otra actividad profesional que exige la mayor parte de su disponibilidad. Alternativamente, una actividad que se ofrece todos los días, o al menos de lunes a viernes, puede ser característica de los proveedores que consideran Airbnb como un canal de distribución de su principal actividad profesional.

Además, comprobamos si proveedores de la plataforma Airbnb ofrecían sus actividades a través de otras plataformas del entorno de la EC. Esto se llevó a cabo buscando en Internet las actividades que ofertaban. Debido al tiempo que ello implicaba, esta búsqueda se limitó a la región de las Islas Canarias.

En esta etapa de la metodología, buscamos también analizar, por medio de entrevistas semiestructuradas presenciales, los motivos que llevaban a ofrecer actividades turísticas en la plataforma Airbnb. No pudimos hacerlo con proveedores no profesionales, ya que la plataforma Airbnb no permite contactar con los anfitriones con fines de investigación y, en general, este tipo de proveedores no disponen de una página web u otra forma de ponerse en contacto con los mismos. En el caso de los proveedores profesionales, elegimos una muestra de treinta empresas y profesionales. Contactamos con ellos a través de su página web y les solicitamos una entrevista. De todos los contactados, quince aceptaron ser entrevistados. La duración promedio de las entrevistas, que se realizaron durante abril y mayo de 2019, fue de 20 minutos.

El objetivo de las entrevistas era explorar sus motivaciones para incluir su oferta en una plataforma de EC, el tipo de oferta que hacían, la utilidad de hacerlo a través de este tipo de plataformas, el perfil de los clientes que conseguían, y una comparación de los mismos con los recibidos a través de otras plataformas B2C.

\section{Resultados}

\subsection{Tipos de actividades turísticas que se ofertan en la EC}

En los cuatro destinos turísticos explorados se encontraron un total de 730 actividades turísticas (Tabla 4). Los talleres fueron los más frecuentes $(31,73 \%)$ seguidos de excursiones y paseos $(22,88 \%)$ y tours temáticos $(13,84 \%)$. Los destinos mostraron diferencias significativas en la oferta de actividades $\left(\chi^{2}=161,69 ; p=0.00\right)$. En Canarias, las actividades deportivas $(13,37 \%)$ y de enseñanza de deportes $(12,83 \%)$, respectivamente, resultaron más frecuentes que los tours temáticos (3,74\%). En Madrid, estos últimos $(27,38 \%)$, la gastronomía (11,31\%) y los tours arquitectónicos e históricos $(11,31 \%)$ resultaron más frecuentes que las excursiones y paseos $(7,14 \%)$. 
Tabla 4: Tipos de actividades turísticas ofrecidas en las regiones analizadas

\begin{tabular}{lrrrrr}
\hline \multicolumn{1}{c}{ Categorías de actividades } & Baleares & Madrid & Canarias & Cataluña & \multicolumn{1}{c}{ Total } \\
\hline Tours arquitectónicos/históricos & $6,90 \%$ & $11,31 \%$ & $3,21 \%$ & $9,72 \%$ & $8,08 \%$ \\
Tours temáticos & $9,20 \%$ & $27,38 \%$ & $3,74 \%$ & $13,98 \%$ & $13,84 \%$ \\
Excursiones/paseos & $33,33 \%$ & $7,14 \%$ & $27,81 \%$ & $25,69 \%$ & $22,88 \%$ \\
Talleres & $33,33 \%$ & $30,95 \%$ & $32,09 \%$ & $30,56 \%$ & $31,73 \%$ \\
Deportes & $8,05 \%$ & $2,98 \%$ & $13,37 \%$ & $4,17 \%$ & $6,71 \%$ \\
Enseñanza de deportes & $1,15 \%$ & - & $12,83 \%$ & $2,43 \%$ & $4,38 \%$ \\
Visitas & $3,45 \%$ & $3,57 \%$ & $4,28 \%$ & $1,04 \%$ & $2,74 \%$ \\
Gastronomía & $3,45 \%$ & $11,31 \%$ & $2,14 \%$ & $9,38 \%$ & $7,26 \%$ \\
Música/baile & $1,15 \%$ & $4,17 \%$ & $0,53 \%$ & $2,78 \%$ & $2,33 \%$ \\
Socialización & - & $1,19 \%$ & - & $0,35 \%$ & $0,41 \%$ \\
\hline Total de actividades & 87 & 168 & 187 & 288 & 730 \\
\hline
\end{tabular}

Fuente: Elaboración propia

Tanto en el alojamiento colaborativo como en el hotelero, el número de valoraciones por parte de los clientes se ha usado como proxy de la demanda (Wang y Nicolau, 2017; Ye, Law y Gu, 2009). La Tabla 5 recoge datos sobre las valoraciones de clientes de las actividades turísticas. En términos absolutos, los tres tipos de actividades más ofertadas también son las que tienen más demanda (i.e., más valoraciones), pero con las excursiones y paseos en primer lugar en lugar de los talleres. La mayoría de las actividades han tenido demanda (77,95\% tienen al menos una calificación). En términos relativos, las actividades de las categorías de música y danza, gastronomía, tours temáticos y tours arquitectónicos e históricos, respectivamente, son las que tienen una mayor aceptación. Más del 80\% de estas se han llevado a cabo. Los clientes están realmente satisfechos con todas las actividades turísticas. Exceptuando la categoría socialización (que cuenta únicamente con dos actividades con valoraciones), la media de las valoraciones de las otras actividades, en una escala de cinco puntos, es siempre superior a 4,74.

Tabla 5: Demanda de las actividades en base al número de valoraciones1 y puntuación media

\begin{tabular}{|c|c|c|c|c|c|c|}
\hline Categorías de actividades & $\begin{array}{c}\text { Total de } \\
\text { valoraciones }\end{array}$ & Media $^{2}$ & $\begin{array}{l}\text { Desv. } \\
\text { típica }^{2}\end{array}$ & $\begin{array}{c}\% \\
\text { valoradas }\end{array}$ & $\begin{array}{c}\text { Puntuación } \\
\text { media }\end{array}$ & $\begin{array}{c}\text { Desv. } \\
\text { típica } \\
\text { puntuac. }\end{array}$ \\
\hline Tours arquitectónicos/históricos & 2.461 & 49,22 & 69,34 & $84,75 \%$ & 4,84 & 0,21 \\
\hline Tours temáticos & 4.038 & 45,89 & 111,10 & $87,13 \%$ & 4,85 & 0,27 \\
\hline Excursiones/paseos & 8.025 & 64,20 & 132,32 & $74,85 \%$ & 4,86 & 0,21 \\
\hline Talleres & 7.746 & 43,76 & 138,31 & $77,29 \%$ & 4,90 & 0,26 \\
\hline Deportes & 671 & 22,37 & 38,13 & $61,22 \%$ & 4,89 & 0,22 \\
\hline Enseñanza de deportes & 288 & 13,10 & 16,98 & $68,75 \%$ & 4,74 & 0,59 \\
\hline Visitas & 242 & 20,17 & 22,45 & $60,00 \%$ & 4,91 & 0,14 \\
\hline Gastronomía & 2.926 & 62,26 & 105,93 & $88,68 \%$ & 4,89 & 0,15 \\
\hline Música/baile & 1.100 & 68,75 & 108,48 & $94,12 \%$ & 4,74 & 0,21 \\
\hline Socialización & 450 & 225,00 & 312,54 & $66,67 \%$ & 4,00 & 1,05 \\
\hline Total & 27.947 & 49,12 & 117,65 & $77,95 \%$ & 4,86 & 0,27 \\
\hline
\end{tabular}

${ }^{1}$ Basado en actividades con al menos una valoración (569/730: 77,95\%).

${ }^{2}$ Media y desviación típica del número de valoraciones por actividad.

${ }^{3}$ Porcentaje de actividades en cada categoría con al menos una valoración.

Fuente: Elaboración propia

Las actividades turísticas tienen una duración media de tres horas (media $=3,01$, dt. $=2,00$ ). Concretamente, el 75\% duraban tres horas y media o menos. La mayoría de las actividades se dirigían 
a grupos de aproximadamente ocho clientes (media $=7,81$, dt. $=5,02$ ). En particular, el $75 \%$ de estas se basaba en grupos de diez o menos consumidores. El precio promedio por persona era 51,35 euros (dt. $=63,30)$, teniendo el $75 \%$ de las actividades un precio de 60 euros o inferior. La correlación entre el precio por persona y el número de valoraciones (i.e., la demanda) fue baja $(0,044)$.

\subsection{Solapamiento entre la oferta de actividades turísticas de la EC con la oferta de las empresas y profesionales turísticos}

Como se explicó anteriormente, para comparar la oferta de actividades turísticas de la EC con la oferta de las empresas turísticas, se analizaron dos plataformas B2C: GetYourGuide y Viator. El análisis de ambas reveló algunas diferencias. Primero, hay cinco tipos de actividades que no se ofrecen a través de Airbnb: venta de tickets, servicios de transfer, excursiones en vehículos, alquiler de vehículos o equipos, y paquetes de excursiones y alojamiento. La Tabla 6 recoge ejemplos de actividades en estas categorías.

Tabla 6: Actividades ofrecidas solo en plataformas B2C

\begin{tabular}{ll}
\hline \multicolumn{1}{c}{ Categorías } & \multicolumn{1}{c}{ Descripción } \\
\hline Venta de tickets & Entradas para el Museo del Prado. \\
Servicios de transfer & Transfer al aeropuerto de Barcelona. \\
Excursiones en vehículos & Tour a las ciudades de Toledo y Segovia. \\
Alquiler de vehículos o equipos & Alquiler de bicicletas eléctricas en Madrid. Alquiler de \\
Paquetes de excursiones y alojamiento & kayaks en Gran Canaria. \\
\hline
\end{tabular}

Fuente: Elaboración propia a partir de GetYourGuide y Viator.

Las tablas 7 y 8 muestran que estas actividades representan el 38,79\% y el 41,39\% de la oferta total en GetYourGuide y Viator, respectivamente. Además, la oferta de excursiones en vehículos es la segunda actividad más importante en GetYourGuide (24,99\%) y la más importante en Viator (23,48\%). En ambas plataformas, esta actividad también es, con mucho, la más importante en las Islas Baleares y las Islas Canarias (27,20\% y 38,72\% en GetYourGuide y $21,50 \%$ y $22,83 \%$ en Viator).

Tabla 7: Tipos de actividades turísticas ofrecidas en GetYourGuide

\begin{tabular}{|c|c|c|c|c|c|}
\hline Categorías de actividades & Baleares & Madrid & Canarias & Cataluña & Total \\
\hline Tours arquitectónicos/históricos & $15,20 \%$ & $41,92 \%$ & $11,79 \%$ & $39,33 \%$ & $31,92 \%$ \\
\hline Tours temáticos & $3,20 \%$ & $3,28 \%$ & $0,26 \%$ & $4,34 \%$ & $3,09 \%$ \\
\hline Excursiones/paseos & $11,20 \%$ & $2,02 \%$ & $17,95 \%$ & $4,84 \%$ & $7,63 \%$ \\
\hline Talleres & - & - & $0,26 \%$ & $0,25 \%$ & $0,17 \%$ \\
\hline Deportes & $14,40 \%$ & $0,76 \%$ & $7,69 \%$ & $0,62 \%$ & $3,26 \%$ \\
\hline Enseñanza de deportes & $4,00 \%$ & - & $7,69 \%$ & $0,12 \%$ & $2,10 \%$ \\
\hline Visitas & $2,40 \%$ & $13,89 \%$ & $1,28 \%$ & $9,55 \%$ & $8,15 \%$ \\
\hline Gastronomía & $1,60 \%$ & $2,27 \%$ & $0,51 \%$ & $3,35 \%$ & $2,33 \%$ \\
\hline Música/baile & $3,20 \%$ & $2,27 \%$ & $0,77 \%$ & $2,73 \%$ & $2,21 \%$ \\
\hline Socialización & $0,80 \%$ & $0,25 \%$ & - & $0,50 \%$ & $0,35 \%$ \\
\hline Venta de tickets & $7,20 \%$ & $2,27 \%$ & $7,18 \%$ & $4,84 \%$ & $4,95 \%$ \\
\hline Servicios de transfer & $4,00 \%$ & $4,04 \%$ & $4,10 \%$ & $1,99 \%$ & $3,09 \%$ \\
\hline Excursiones en vehículos & $27,20 \%$ & $14,65 \%$ & $38,72 \%$ & $23,08 \%$ & $24,99 \%$ \\
\hline Alquiler de vehículos o equipos & $5,60 \%$ & $0,76 \%$ & $1,79 \%$ & $2,48 \%$ & $2,15 \%$ \\
\hline Paquetes de excursiones y alojamiento & - & $11,62 \%$ & - & $1,99 \%$ & $3,61 \%$ \\
\hline Total de actividades & 125 & 396 & 390 & 806 & 1.717 \\
\hline
\end{tabular}

Fuente: Elaboración propia 
Tabla 8: Tipos de actividades turísticas ofrecidas en Viator

\begin{tabular}{lrrrrr}
\hline \multicolumn{1}{c}{ Categorías de actividades } & Baleares & Madrid & Canarias & Cataluña & \multicolumn{1}{c}{ Total } \\
\hline Tours arquitectónicos/históricos & $15,89 \%$ & $28,71 \%$ & $7,11 \%$ & $34,31 \%$ & $23,43 \%$ \\
Tours temáticos & $3,18 \%$ & $3,54 \%$ & $4,25 \%$ & $4,59 \%$ & $4,04 \%$ \\
Excursiones/paseos & $11,03 \%$ & $2,56 \%$ & $18,58 \%$ & $4,67 \%$ & $8,54 \%$ \\
Talleres & $0,19 \%$ & $1,57 \%$ & $0,59 \%$ & $1,58 \%$ & $1,14 \%$ \\
Deportes & $8,60 \%$ & $3,24 \%$ & $14,82 \%$ & $2,08 \%$ & $6,52 \%$ \\
Enseñanza de deportes & $3,18 \%$ & - & $12,94 \%$ & $0,65 \%$ & $3,97 \%$ \\
Visitas & $2,99 \%$ & $8,46 \%$ & $1,68 \%$ & $7,47 \%$ & $5,64 \%$ \\
Gastronomía & $3,18 \%$ & $2,36 \%$ & $1,98 \%$ & $5,10 \%$ & $3,34 \%$ \\
Música/baile & $1,12 \%$ & $2,56 \%$ & $0,40 \%$ & $2,44 \%$ & $1,77 \%$ \\
Socialización & - & $0,20 \%$ & $0,30 \%$ & $0,29 \%$ & $0,23 \%$ \\
Venta de tickets & $4,30 \%$ & $2,65 \%$ & $7,02 \%$ & $3,88 \%$ & $4,42 \%$ \\
Servicios de transfer & $14,95 \%$ & $9,54 \%$ & $4,64 \%$ & $7,32 \%$ & $8,24 \%$ \\
Excursiones en vehículos & $21,50 \%$ & $27,63 \%$ & $22,83 \%$ & $21,68 \%$ & $23,48 \%$ \\
Alquiler de vehículos o equipos & $9,72 \%$ & $0,39 \%$ & $2,27 \%$ & $1,44 \%$ & $2,50 \%$ \\
Paquetes de excursiones y alojamiento & $0,19 \%$ & $6,59 \%$ & $0,59 \%$ & $2,51 \%$ & $2,75 \%$ \\
\hline Total de actividades & 535 & 1.017 & 1.012 & 1.393 & 3.957 \\
\hline
\end{tabular}

Fuente: Elaboración propia

En cuanto a las actividades comunes entre Airbnb y las plataformas B2C, estas presentan algunas diferencias. En las plataformas B2C, los tours arquitectónicos e históricos son el tipo de actividad más importante, mientras que ocupa el cuarto lugar en Airbnb. En las plataformas B2C, los talleres son el tipo de actividad menos frecuente o el segundo menos frecuente $(0,17 \%$ en GetYourGuide y 1,14\% en Viator). Por el contrario, son la actividad más importante en Airbnb (31,73\%). Además, los tours temáticos son el tercer tipo de actividad más frecuente en Airbnb, mientras que este tipo de actividad se encuentra en la quinta posición (GetYourGuide) y la séptima posición (Viator) en las plataformas B2C.

\subsection{Atributos sobre los que se fundamentan las actividades turísticas de la EC}

Los resultados del análisis de contenido de las autodescripciones de los proveedores no revelaron un perfil predominante de proveedor. Surgieron cuatro tipos: aquellos que resaltan características no profesionales, los que basan sus descripciones en características profesionales, los que combinan los dos casos anteriores, y aquellos que destacan sus logros académicos. Se comentan a continuación.

El 25,92\% de los proveedores de actividades mencionan solo características no profesionales en sus perfiles. Resaltan su pasión por la actividad (e.g., quieren compartir su pasión por la ciudad en la que viven; su pasión por los coches antiguos), otras actitudes similares, como el placer que sienten por la actividad en cuestión (e.g., quieren compartir el placer de navegar con otros), ser un amante de algo (e.g., ser un amante de la cultura y de la historia; ser un apasionado de la gastronomía), o el deseo de compartir algo (e.g., experiencia musical).

El 20,47\% de los proveedores destacan características exclusivamente profesionales en sus perfiles. Resaltaron la experiencia profesional (e.g., años de experiencia como guía en diferentes áreas; trabajar como profesor de inglés para ejecutivos de empresas), acreditaciones profesionales (e.g., capitán profesional; fotógrafo profesional; guía profesional), ser propietario de una empresa especializada (e.g., haber abierto un negocio de tours guiados; ser fundador de un negocio de alquiler de bicicletas), y trabajar para una empresa especializada (e.g., gerente de una empresa de eventos; trabajar para una empresa dedicada a actividades musicales).

El 25,92\% de los proveedores incluyeron en sus perfiles una combinación de características no profesionales y profesionales (e.g., instructor de surf y entusiasta de los deportes extremos). 
El 9,72\% de los proveedores destacaron sus credenciales académicas (e.g., licenciado en administración de empresas y turismo; licenciado en derecho y MBA; doctorado en historia española). Estos perfiles aparecieron en actividades en las que también se resaltaban características no profesionales.

Finalmente, el 17,97\% de los proveedores tenían un perfil que no nos permitía clasificarlos en ninguna de las categorías anteriores. Esto se debió a varias razones. En algunos casos, la información incluida fue muy corta y no contenía ninguna característica relevante para este análisis (e.g., solo la edad y el sexo), mientras que en otros la información se completó incorrectamente (e.g., información sobre la ubicación de las actividades ofrecidas, que debería estar en otra parte del perfil).

\subsection{Tipos de proveedores y actividades predominantes en la EC}

Las 730 actividades turísticas de Airbnb eran ofrecidas por 567 proveedores. Por lo tanto, estos ofertaban un promedio de 1,29 actividades (dt. $=0,68$ ). Un 79,72\% ofrecía solo una actividad, un $14,46 \%$ dos, un $4,41 \%$ tres, un $0,53 \%$ cuatro, un $0,71 \%$ cinco y un $0,18 \%$ siete actividades.

La Tabla 9 muestra la disponibilidad temporal de las actividades turísticas ofertadas en Airbnb Experiences. El 22,74\% se ofrecían diariamente. Este tipo de oferta podría ser propia de los proveedores profesionales y también de aquellos que buscan una actividad profesional en Airbnb. Las actividades de enseñanza deportiva fueron las que más destacaron en este tipo de frecuencia (43,75\%), mientras que los tours arquitectónicos e históricos fueron las que menos (13,56\%).

No resultaba habitual ofrecer actividades turísticas solo los fines de semana o de lunes a viernes (ambos 4,25\%). Incluso ofertarlas tres días, o uno o dos días, a la semana no era común (ambas opciones están por debajo del 20\%). Mayoritariamente, la disponibilidad de las actividades turísticas no seguía un patrón regular $(34,11 \%)$ reflejado en la columna denominada irregular. Se incluyen en esta categoría las actividades cuya oferta no era sistemática, como lunes, martes, miércoles y viernes una semana, solo el viernes la semana siguiente, lunes y viernes las dos semanas siguientes, ninguna oferta la semana siguiente, y lunes, miércoles y viernes la siguiente semana. También incluye aquellas actividades con muy baja disponibilidad (es decir, dos días al mes). Este tipo de oferta podría ser característico de los proveedores que tienen otras actividades profesionales y que usan la plataforma solo cuando pueden. La disponibilidad de las actividades no mostró diferencias significativas entre las actividades de los proveedores que ofrecieron solo una actividad y las actividades de aquellos que ofrecieron más de una ( $\left.\chi^{2}: 6.2338 ; \mathrm{p}>0.05\right)$.

Tabla 9: Disponibilidad de las actividades en Airbnb

\begin{tabular}{lrrrrrrr}
\hline \multicolumn{1}{c}{$\begin{array}{c}\text { Categorías de } \\
\text { actividades }\end{array}$} & $\begin{array}{c}\text { Todos } \\
\text { los días }\end{array}$ & $\begin{array}{c}\text { Solo } \\
\text { fines de } \\
\text { semana }\end{array}$ & $\begin{array}{c}\text { De } \\
\text { lunes a } \\
\text { viernes }\end{array}$ & $\begin{array}{c}\text { Tres } \\
\text { días }\end{array}$ & $\begin{array}{c}\text { Uno o } \\
\text { dos días }\end{array}$ & Irregular & Casos \\
\hline $\begin{array}{l}\text { Tours arquitectónicos/ } \\
\text { históricos }\end{array}$ & $13,56 \%$ & $1,69 \%$ & $6,78 \%$ & $25,42 \%$ & $18,64 \%$ & $33,90 \%$ & 59 \\
Tours temáticos & $22,77 \%$ & $2,97 \%$ & $5,94 \%$ & $22,77 \%$ & $14,85 \%$ & $30,69 \%$ & 101 \\
Excursiones/paseos & $23,95 \%$ & $2,99 \%$ & $4,19 \%$ & $13,77 \%$ & $15,57 \%$ & $39,52 \%$ & 167 \\
Talleres & $22,71 \%$ & $4,37 \%$ & $4,80 \%$ & $16,16 \%$ & $16,16 \%$ & $35,81 \%$ & 229 \\
Deportes & $18,37 \%$ & $4,08 \%$ & - & $18,37 \%$ & $28,57 \%$ & $30,61 \%$ & 49 \\
Enseñanza de deportes & $43,75 \%$ & $12,50 \%$ & - & $3,13 \%$ & $9,38 \%$ & $31,25 \%$ & 32 \\
Visitas & $15,00 \%$ & $5,00 \%$ & - & $20,00 \%$ & $30,00 \%$ & $30,00 \%$ & 20 \\
Gastronomía & $20,75 \%$ & $9,43 \%$ & $5,66 \%$ & $11,32 \%$ & $32,08 \%$ & $20,75 \%$ & 53 \\
Música/baile & $29,41 \%$ & - & - & $5,88 \%$ & $17,65 \%$ & $47,06 \%$ & 17 \\
Socialización & $33,33 \%$ & - & - & $33,33 \%$ & $33,33 \%$ & & -3 \\
\hline Total & $22,74 \%$ & $4,25 \%$ & $4,25 \%$ & $16,44 \%$ & $18,22 \%$ & $34,11 \%$ & 730 \\
\hline
\end{tabular}

Fuente: Elaboración propia

Como explicamos en la metodología, analizamos las 187 actividades disponibles en las Islas Canarias con más detalle. Estas eran ofertadas por 141 proveedores diferentes. El 64,52\% por empresas o profe- 
sionales locales que representaban el $51,77 \%$ del total de proveedores. La mayoría de estos proveedores profesionales ofrecían las mismas actividades fuera del entorno de la EC (82,19\%). El 56,15\% usaba su propia página web para promocionar y vender sus actividades. También se apoyaban en diferentes redes sociales para promocionar sus productos: Facebook (31,02\%), TripAdvisor (12,83\%), Instagram $(5,88 \%)$ y LinkedIn (5,35\%). El otro 17,81\% solo usaba Airbnb.

Las actividades turísticas ofrecidas por profesionales consistían principalmente en excursiones y paseos $(27,63 \%)$, enseñanza de deportes $(27,63 \%)$, talleres $(18,24 \%)$ y tours temáticos $(11,84 \%)$. Estos últimos se caracterizaban incluir activos físicos que un aficionado no puede permitirse fácilmente: botes, canoas y bicicletas para excursiones; tablas de surf y material de buceo para la enseñanza deportiva; espacio para aprender una actividad en el caso de los talleres (e.g., yoga o flamenco); y medios de transporte e instalaciones para los tours temáticos.

\subsection{Razones para usar la EC para ofrecer actividades turísticas}

Como se describe en la metodología, en la segunda etapa seguimos un enfoque cualitativo basado en entrevistas. Entrevistamos a quince proveedores profesionales para comprender sus motivaciones sobre el uso de plataformas de EC. Solo uno tenía empleados (cuatro), mientras que los otros eran trabajadores autónomos o socios de una pequeña empresa sin empleados. Excepto un proveedor que había comenzado en Airbnb hace solo tres meses, los otros usaban esta plataforma desde hace más de un año. Uno de los proveedores solo ofertaba su actividad a través de Airbnb. La promocionaba a través de Facebook e Instagram, pero las transacciones comerciales solo se hacían en Airbnb. Estaba lanzando su pequeño negocio apoyándose en esta plataforma. Los otros proveedores utilizaban, además de Airbnb, otros canales de comercialización digital, como sus propias páginas web, plataformas B2C (GetYourGuide, Viator, Civitatis, Urlaub-kreativ, TripAdvisor y Vimotions), así como sus oficinas físicas. Una proveedora también usaba la plataforma de EC Etsy para sus creaciones (era una artesana que ofrecía cursos de manualidades y vendía sus creaciones en línea).

Casi todos los proveedores entrevistados promocionaban sus actividades mediante las redes sociales Facebook e Instagram. Dos proveedores ofrecían actividades a través de Airbnb que eran una adaptación de las actividades que comercializaban a través de los otros canales. Uno la había modificado porque Airbnb no admite actividades de alto riesgo y el otro ofertaba una versión menos especializada de su actividad. Los proveedores restantes ofertaban la misma actividad en todos sus canales de comercialización.

Los proveedores usaban Airbnb por motivos económicos, siendo la plataforma una forma de obtener ingresos. Excepto por el proveedor que solo comercializaba su actividad a través de Airbnb, los ingresos obtenidos mediante la misma representaban entre el 10\% y el 50\% de los ingresos totales de los proveedores. Para ellos Airbnb era una forma de obtener un tipo diferente de clientes, en comparación con los que conseguían a través de otros canales de comercialización. Estas diferencias eran en términos de nacionalidad (extranjeros en lugar de locales o nacionales, o extranjeros de nacionalidades específicas que no llegaban por otros canales), edad (personas más jóvenes que las que llegaban por otros canales) y tipo de cliente (en el sentido de que Airbnb les lleva a clientes menos especializados). Ninguno de los proveedores comentó las razones teóricamente asociadas a los intercambios en la EC (e.g., el placer de interactuar con otras personas). La mitad de los proveedores nunca se había hospedado en un alojamiento propio de la EC y ninguno de ellos actuaba como anfitrión de una vivienda en plataformas de EC.

Los proveedores comentaban que Airbnb permite una relación más estrecha con los clientes. Pueden usar el correo electrónico y el teléfono para resolver directamente sus consultas y adaptar la actividad a sus intereses. Esto era poco frecuente en otros canales de comercialización, en los que apenas se producen interacciones directas con los clientes. También manifestaban que Airbnb aumenta la visibilidad de sus actividades. Algunos de ellos destacaron que la plataforma recomienda automáticamente sus actividades a clientes alojados en viviendas colaborativas cercanas. Dos proveedores también mencionaron que la comisión de Airbnb era más baja que la que cobran otras plataformas, aunque uno de los proveedores entrevistados declaró que la otra plataforma que usaba era gratuita.

\section{Discusión}

Por su naturaleza, las actividades turísticas de la EC podrían escapar de la influencia de los responsables de los destinos turísticos. Este es un aspecto que no se ha tenido en cuenta en la literatura sobre la EC en el sector turístico. Sin embargo, este tipo de actividades no deberían ser motivo de preocupación, ya que el grado de satisfacción de los consumidores con las mismas es muy elevado. No 
obstante, estas valoraciones deben ser interpretadas con cautela, dado que la literatura ya ha descrito que hay un efecto de inflación de la reputación en la EC (Bulchand-Gidumal \& Melián-González, 2019). Por otro lado, aunque distintos autores afirman que estas actividades pueden incrementar el atractivo de los destinos, ya que los visitantes quieren conocer a locales que brindan autenticidad, es necesario tener en cuenta que estos no son los principales proveedores. Creemos que este resultado contribuye a clarificar, por lo menos en el ámbito del turismo, quiénes están detrás de la oferta turística en el ámbito de la EC. Así, este hallazgo confirma los descritos en la revisión de la literatura y que se ciñen básicamente al alojamiento colaborativo.

En relación a lo anterior, creemos que no sería demasiado arriesgado afirmar que al menos el 34\% de las actividades turísticas son ofrecidas por proveedores no profesionales. La cifra se basa en la forma irregular en que se ofrecen estos productos. Es difícil que profesionales oferten sus actividades de manera tan asistemática. Esta cantidad de actividades no profesionales podría ser ligeramente mayor si se añaden las actividades ofrecidas solo los fines de semana (4,25\%). Pero incluso así, esto implica que las actividades turísticas que ofrecen los profesionales prevalecerían en la EC, representando alrededor del $60 \%$ o más. La medición más directa de la cantidad de actividades profesionales en las Islas Canarias $(64,52 \%)$ apoya esta cifra. En esta región el 51,77\% de los proveedores de eran pequeñas empresas o profesionales especializados

Aunque similares, las actividades turísticas de la EC y las de las plataformas B2C tienen diferencias importantes. Considerando los tipos de actividades, aproximadamente el $60 \%$ de las ofertas de ambos tipos de plataformas coinciden. Este resultado coincide con los estudios que muestran que el alojamiento colaborativo y el hotelero pueden ser opciones equivalentes desde el punto de vista de los clientes. Sin embargo, las plataformas B2C ofrecen actividades diferentes que se basan en equipos costosos, como vehículos para el transporte de turistas o su alquiler. Otra diferencia relevante es la gran importancia de los talleres en la plataforma de EC y la poca importancia que tienen en las plataformas B2C. Esto podría reflejar que Airbnb depende de particulares y/o pequeños profesionales que pueden proponer actividades más especializadas y actividades basadas en sus habilidades personales. Por el contrario, las plataformas B2C dependen más de actividades altamente estandarizadas, como tours arquitectónicos o históricos, que fue el tipo de actividad más frecuente entre aquellas que eran comunes con Airbnb.

Las actividades turísticas en la EC no son sinónimo de proveedores apasionados. Esto contradice mucha de la retórica en la que se sustenta buena parte de la literatura divulgativa sobre EC. En cuanto a las publicaciones académicas, aquellos que afirman que la EC puede ser una fuente de experiencias auténticas se refieren a este tipo de perfil de proveedor. Sin embargo, solo el 25,92\% de los perfiles de proveedores se basan en este tipo de atributos. Si también consideramos aquellos que combinan la pasión y atributos profesionales, esta cantidad aumenta, pero no pasa de la mitad de los perfiles. En cualquier caso, todavía hay una cantidad significativa de perfiles de proveedores $(46,39 \%)$ que no se basan en atributos relacionados con la pasión o entusiasmo por su actividad. En cuanto a las plataformas B2C, no se basan en este tipo de perfiles.

La alta presencia de profesionales estimada puede explicarse por los beneficios que los proveedores encuentran en Airbnb. Ello coincide con las opiniones de numerosos autores respecto a que buena parte de la EC, aquella más importante desde el punto de vista de número de usuarios, es un mercado económico, con transacciones básicamente comerciales. Las entrevistas reflejaron que los proveedores profesionales de la EC han encontrado un canal de distribución adicional para sus actividades habituales. La mayoría de ellos vendían las mismas actividades, o actividades muy similares, que ya ofrecían en otros canales de comercialización. Estos proveedores afirmaban que a través de Airbnb estaban llegando a un segmento diferente de clientes. Este nuevo canal puede ser importante para algunos de ellos, como lo demuestran los dos casos que obtenían la mitad de sus ingresos a través de Airbnb. Los proveedores profesionales entrevistados no mencionaron factores relacionados con la pasión como motivos para ofrecer las actividades. Además, ninguno de ellos parecía ser entusiasta de la EC. Sin embargo, sí comentaron que Airbnb les permitía ofrecer servicios más personalizados, ya que la plataforma fomenta una relación directa con los clientes, una vez que estos han reservado y pagado la actividad.

\section{Conclusiones}

Existe una notable coincidencia (60\%) entre los tipos de actividades turísticas que se ofrecen en las plataformas Airbnb y dos de las plataformas más relevantes de reserva de actividades B2C (GetYourGuide y Viator). Al mismo tiempo, hay dos diferencias importantes. Primero, las plataformas B2C ofrecen 
actividades diferentes basadas en recursos costosos, como vehículos para el transporte y alquiler. En segundo lugar, y con respecto a las actividades comunes, la mayor presencia de talleres en Airbnb sugiere que la oferta en esta plataforma se basa en proveedores más especializados. Estas coincidencias y diferencias en el campo de las actividades turísticas se asemejan a los hallazgos existentes respecto al alojamiento colaborativo y los hoteles: aunque la evidencia sugiere una gran similitud entre ambos tipos de alojamiento, hay espacio para diferentes tipos de servicios tanto en la EC como en la oferta hotelera tradicional.

Las actividades turísticas de la EC tienen una gran demanda (el 77,95\% cuentan con al menos una valoración, por lo que seguramente en torno del $80 \%$ han sido contratadas en al menos una ocasión). Una característica de la EC es que la oferta supera con creces la demanda. En otros campos de la EC, los porcentajes de servicios que realmente se han demandado son significativamente más bajos (De Groen, Maselli y Fabo, 2016; Melián-González y Bulchand-Gidumal, 2018b).

También, y en línea con lo que es común en la EC (Bulchand-Gidumal y Melián-González, 2019), los consumidores han demostrado estar extremadamente satisfechos con su experiencia. Por lo tanto, los responsables de las DMO no deberían preocuparse por las percepciones de los turistas respecto a este tipo de servicios.

Como se ha visto, la oferta de la EC en el ámbito de las actividades turísticas nace de la iniciativa de un amplio conjunto de profesionales. Los responsables de las DMO pueden encontrar en la misma una fuente de información valiosa de lo que ocurre en sus destinos, tanto desde el punto de vista de la oferta como de la demanda. Encontrar esta información en la EC es más sencillo que hacerlo en el entorno económico tradicional.

Por último, mencionar que los profesionales pueden encontrar una oportunidad en las actividades turísticas de la EC. Parece que hay una mayoría de actividades ofrecidas por los mismos (60\%). Mayoritariamente ofrecen las mismas actividades que en otros canales de distribución. Estas necesitan recursos físicos que un aficionado encontraría difícil de costear. Los motivos por los que usan Airbnb son económicos. Es cierto que la mitad de los proveedores destacan la pasión que sienten por las actividades que ofrecen, pero una cantidad similar también se apoyaba en características profesionales tradicionales. Todo ello coincide con la afirmación de Airbnb respecto a que los proveedores son apasionados de sus actividades y emprendedores.

\section{Limitaciones}

Como primer análisis de la EC en el campo de las actividades turísticas, este estudio no está exento de ciertas limitaciones. Primero, se basa en una muestra limitada: cuatro destinos turísticos españoles, para los que se descargaron todas las actividades; una región española, que se analizó en profundidad; y quince profesionales que fueron entrevistados. Esto dificulta la generalización de los hallazgos. En segundo lugar, el hecho de que la cifra de actividades ofrecidas por proveedores profesionales se determinó a través de un proxy: la disponibilidad de la actividad en los siguientes tres meses. Una medida directa sería más válida. Por último, nos hubiera gustado realizar entrevistas con proveedores no profesionales, pero no pudimos obtener los datos de contacto de una muestra lo suficientemente grande como para que los resultados fueran mínimamente relevantes.

\section{Bibliografía}

Aloisi, A. 2016. Commoditized workers. Case study research on labour law issues arising from a set of 'on-demand/gig economy' platforms. Comparative Labor Law \& Policy Journal, 37(3), 653-684.

Apte, U. M., \& Davis, M. M. 2019. Sharing Economy Services: Business Model Generation. California Management Review, 61(2), 104-131.

Batle, J., Garau-Vadell, J. B., \& Orfila-Sintes, F. 2019. Are locals ready to cross a new frontier in tourism? Factors of experiential P2P orientation in tourism. Current Issues in Tourism. Advanced online publication. https://doi.org/10.1080/13683500.2019.1604639.

Beerli, A., \& Martin, J. D. 2004. Factors influencing destination image. Annals of Tourism Research, 31(3), 657-681.

Benítez-Aurioles, B. 2019. Is Airbnb bad for hotels? Current Issues in Tourism, 1-4. Advanced online publication. https://doi.org/10.1080/13683500.2019.1646226. 
Berger, T., Chen, C., \& Frey, C. B. 2018. Drivers of disruption? Estimating the Uber effect. European Economic Review, 110, 197-210.

Blal, I., Singal, M., \& Templin, J. 2018. Airbnb's effect on hotel sales growth. International Journal of Hospitality Management, 73, 85-92.

Botsman, R., \& Rogers, R. 2011. What's mine is yours: how collaborative consumption is changing the way we live. London: Collins.

Bryon, J. 2012. Tour guides as storytellers-from selling to sharing. Scandinavian Journal of Hospitality and Tourism, 12(1), 27-43.

Buckley, R. 2007. Adventure tourism products: Price, duration, size, skill, remoteness. Tourism Management, 28(6), 1428-1433.

Buhalis, D. 2000. Marketing the competitive destination of the future. Tourism Management, 21(1), $97-116$.

Bulchand-Gidumal, J., \& Melián-González, S. (2017). La revolución de la economía colaborativa [The revolution of the sharing economy]. Madrid: Lid Editorial.

Bulchand-Gidumal, J., Melián-González, S., \& López-Valcárcel, B. G. 2019. Is the sharing economy for all? An answer based on neighbourhoods, types of hosts, and user complaints. In Information and Communication Technologies in Tourism 2019 (pp. 55-66). Springer, Cham.

Bulchand-Gidumal, J., \& Melián-González, S. 2019. Why are ratings so high in the sharing economy? Evidence based on guest perspectives. Current Issues in Tourism. Advanced online publication. https://doi.org/10.1080/13683500.2019.1602597.

Cramer, J., \& Krueger, A. B. 2016. Disruptive change in the taxi business: The case of Uber. American Economic Review, 106(5), 177-82.

Crouch, G. I., \& Ritchie, J. B. 1999. Tourism, competitiveness, and societal prosperity. Journal of Business Research, 44(3), 137-152.

Crouch, G. I., \& Ritchie, J. B. 2000. The competitive destination: A sustainability perspective. Tourism management, 21(1), 1-7.

De Groen, W. P., Maselli, I., \& Fabo, B. 2016. The Digital Market for Local Services: A one night stand for workers? (CEPS Special Report No. 133). Retrieved from https://papers.ssrn.com/sol3/papers. cfm?abstract_id=2766220.

Dredge, D., \& Gyimóthy, S. 2015. The collaborative economy and tourism: Critical perspectives, questionable claims and silenced voices. Tourism Recreation Research, 40(3), 286-302.

Frenken, K., \& Schor, J. 2017. Putting the sharing economy into perspective. Environmental Innovation and Societal Transitions, 23, 3-10.

Gardiner, S. 2018. The new wave of tourism experiences in the sharing economy. Paper presented at the CAUTHE 2018: Get Smart: Paradoxes and Possibilities in Tourism, Hospitality and Events Education and Research.

Guttentag, D. A., \& Smith, S. L. 2017. Assessing Airbnb as a disruptive innovation relative to hotels: Substitution and comparative performance expectations. International Journal of Hospitality Management, 64, 1-10.

Guttentag, D., Smith, S., Potwarka, L., \& Havitz, M. 2018. Why tourists choose Airbnb: a motivation-based segmentation study. Journal of Travel Research, 57(3), 342-359.

Gyódi, K. 2019. Airbnb in European cities: Business as usual or true sharing Economy? Journal of Cleaner Production, 221 (1), 536-551.

Hall, J. V., \& Krueger, A. B. 2015. An analysis of the labor market for Uber's driver-partners in the United States. Retrieved from https://assets.documentcloud.org/documents/1507970/uberstudy.pdf.

Heath, E., \& Wall, G. 1992. Marketing tourism destinations: A strategic planning approach. New York: Wiley.

Ketter, E. 2019. Eating with EatWith: analysing tourism-sharing economy consumers. Current Issues in Tourism, 22(9), 1062-1075.

Kurtzman, J. 2005. Sports tourism categories. Journal of Sport Tourism, 10(1), 15-20.

Li, J., Moreno, A., \& Zhang, D. J. 2015. Agent behavior in the sharing economy: Evidence from Airbnb. Ross School of Business Working Paper Series, 1298. Retrieved from https://pdfs.semanticscholar. org/9cb2/e1ad853d8cec5cb2ccd6eb3df951aa2733ac.pdf

Melián-González, S. 2018. What Do Consumers Appreciate about On-Demand Economy Workers?. Psychosociological Issues in Human Resource Management, 6(2), 29-43. 
Melián-González, S., \& Bulchand-Gidumal, J. 2018a. Análisis de la actividad desarrollada en el turismo colaborativo: el caso Trip4real [An analysis of the activity developed in sharing tourism: The Trip4real case]. Paper presented at AECIT conference 2018, Spain. https://aecit.org/files/congress/19/ papers/171.pdf

Melián-González, S., \& Bulchand-Gidumal, J. 2018b. What type of labor lies behind the on-demand economy? New research based on workers' data. Journal of Management \& Organization. Advanced online publication. https://doi.org/10.1017/jmo.2018.53.

Müller, H., \& Berger, P. 2012. Benchmarking for destination management organizations: the case of Swiss cities and Alpine destination management. Tourism Review, 67(4), 26-39.

Paulauskaite, D., Powell, R., Coca- Stefaniak, J. A., \& Morrison, A. M. 2017. Living like a local: Authentic tourism experiences and the sharing economy. International Journal of Tourism Research, 19(6), 619-628.

Presenza, A., Sheehan, L., \& Ritchie, J. B. 2005. Towards a model of the roles and activities of destination management organizations. Journal of Hospitality, Tourism and Leisure Science, 3(1), 1-16.

Ritchie, J. R. B., \& Crouch, G. I. 2003. The competitive destination: A sustainable tourism perspective. Wallingford, UK: CABI Publishing.

Sainaghi, R. 2006. From contents to processes: versus a dynamic destination management model (DDMM). Tourism Management, 27(5), 1053-1063.

Saldaña, J. 2013. The coding manual for qualitative researchers. California: Sage.

Schor, J. B. 2014. Debating the Sharing Economy. Retrieved from http://www.msaudcolumbia.org/ summer/wp-content/uploads/2016/05/Schor_Debating_the_Sharing_Economy.pdf.

Schor, J. B. 2017. Does the sharing economy increase inequality within the eighty percent?: findings from a qualitative study of platform providers. Cambridge Journal of Regions, Economy and Society, 10(2), 263-279.

Schor, J. B., \& Attwood- Charles, W. 2017. The "sharing" economy: labor, inequality, and social connection on for- profit platforms. Sociology Compass, 11(8), 1-16.

Sundarajaran, A. 2014. Peer-to-Peer Businesses and the Sharing (Collaborative) Economy: Overview, Economic Effects and Regulatory Issues. Written testimony for the hearing titled The Power of Connection: Peer to Peer Businesses, January. Retrieved from http://smbiz.house.gov/uploadedfiles/1-15-2014_revised_sundararajan_testimony.pdf.

Wang, D., \& Nicolau, J. L. 2017. Price determinants of sharing economy based accommodation rental: A study of listings from 33 cities on Airbnb. com. International Journal of Hospitality Management, 62, 120-131.

Ye, Q., Law, R., \& Gu, B. 2009. The impact of online user reviews on hotel room sales. International Journal of Hospitality Management, 28 (1), 180-182.

Zervas, G., Proserpio, D., \& Byers, J. W. 2017. The rise of the sharing economy: Estimating the impact of Airbnb on the hotel industry. Journal of Marketing Research, 54(5), 687-705.

Zhang, T., Bufquin, D., \& Lu, C. 2019. A qualitative investigation of microentrepreneurship in the sharing economy. International Journal of Hospitality Management, 79, 148-157.

\section{Notas}

1 https://www.ine.es/dyngs/INEbase/es/operacion.htm?c=Estadistica_C\&cid=1254736176996\&menu=ultiDatos\&i $\mathrm{dp}=1254735576863$ 